\title{
Article \\ Does an Antibiotic Stewardship Applied in a Pig Farm Lead to Low ESBL Prevalence?
}

\author{
Claudine Fournier ${ }^{1}$ (D), Patrice Nordmann ${ }^{2,3,4}\left(\mathbb{D}\right.$, Olivier Pittet ${ }^{5}$ and Laurent Poirel ${ }^{2,3, *(\mathbb{D})}$ \\ 1 Medical and Molecular Microbiology Unit, Faculty of Science and Medicine, University of Fribourg, \\ 1700 Fribourg, Switzerland; claudine.fournier@unifr.ch \\ 2 INSERM European Unit (IAME, France), University of Fribourg, 1700 Fribourg, Switzerland; \\ patrice.nordmann@unifr.ch \\ 3 Swiss National Reference Center for Emerging Antibiotic Resistance (NARA), University of Fribourg, \\ 1700 Fribourg, Switzerland \\ 4 Institute for Microbiology, University Hospital Centre, 1011 Lausanne, Switzerland \\ 5 Agricultural Institute of the Canton of Fribourg, Grangeneuve, 1725 Posieux, Switzerland; Olivier.Pittet@fr.ch \\ * Correspondence: laurent.poirel@unifr.ch; Tel.: +41-26-300-9582
}

Citation: Fournier, C.; Nordmann, P.; Pittet, O.; Poirel, L. Does an Antibiotic Stewardship Applied in a Pig Farm Lead to Low ESBL

Prevalence? Antibiotics 2021, 10, 574.

https://doi.org/10.3390/

antibiotics10050574

Academic Editor: Susana Ferreira

Received: 19 March 2021

Accepted: 7 May 2021

Published: 13 May 2021

Publisher's Note: MDPI stays neutral with regard to jurisdictional claims in published maps and institutional affiliations.

Copyright: (c) 2021 by the authors. Licensee MDPI, Basel, Switzerland. This article is an open access article distributed under the terms and conditions of the Creative Commons Attribution (CC BY) license (https:// creativecommons.org/licenses/by/ $4.0 /)$.

\begin{abstract}
Background. The aim of the present study was to prospectively evaluate the prevalence of intestinal carriage of colistin-resistant and extended-spectrum $\beta$-lactamase (ESBL)-producing Enterobacterales among pigs from a Swiss farm attending an animal health and antibiotic stewardship program and to determine the associated mechanisms of resistance. Materials/Methods. Eightyone fecal samples were recovered and screened for either $\beta$-lactam-resistant, colistin-resistant, or aminoglycoside-resistant Enterobacterales, using respective screening media. All recovered isolates were tested for antimicrobial susceptibility and their clonal relationship (PFGE and MLST). Plasmid typing was performed by plasmid-based replicon typing (PBRT). Resistance genes were searched by PCR and sequencing. Results. A total of 38 ESBL-producing Escherichia coli and a single ESBLproducing Enterobacter cloacae were recovered from 81 pigs, corresponding to a prevalence of $50 \%$, no other $\beta$-lactamase producer being identified. Among the 38 ESBL-producing E. coli, all belonged to

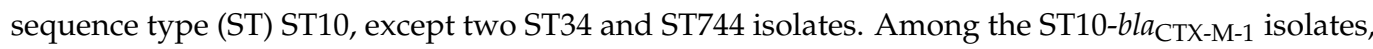
three subclones ( $n=22, n=13$, and $n=1$, respectively) were identified according to the PFGE analysis. The most commonly identified IncI1 plasmid harboring the $b l a_{\mathrm{CTX}-\mathrm{M}-1}$ gene was $143 \mathrm{~kb}$ in size and coharbored other resistance genes. Only three colistin-resistant Enterobacterales isolates were recovered, namely two Klebsiella pneumoniae isolates and a single E. cloacae isolate. Screening for the plasmid-borne $m c r-1$ to $m c r-9$ genes in these three isolates gave negative results. The two K. pneumoniae isolates were clonally related, belonged to ST76, and harbored a truncated $\operatorname{mgr} B$ chromosomal gene being the source of colistin resistance. Conclusion. A high prevalence of fecal carriage of ESBL-producing E. coli was found, being mainly caused by the spread of a clonal lineage within the farm. By contrast, a low prevalence of colistin-resistant Enterobacterales was found.
\end{abstract}

Keywords: ESBL; colistin; mobilized colistin resistance; pigs

\section{Introduction}

Antibiotic resistance is now considered a major public health concern, since it is nowadays on a rising trend, impacting the whole world and leading to difficult-to-treat infections. Moreover, recent perspectives indicate that the problem of antibiotic resistance shall be considered as a One Health issue, since the rising trend observed in human medicine is also observed in veterinary medicine and to a larger extent in the environment, especially among Gram-negatives [1]. Therefore, controlling the spread of multidrug resistance in one of these compartments might influence the others [2].

Food-producing animals, particularly swine, are known to be important reservoirs of antibiotic-resistant Enterobacterales and potential sources of transmission to humans [3,4]. 
In Switzerland, antibiotics, particularly $\beta$-lactams, may be prescribed to swine as therapeutics in case of infections. Hence, the microbiota of swine animals can be subjected to antibiotic selective pressure, and these animals may subsequently be colonized with multiresistant bacteria.

The aim of our study was to prospectively screen pigs from a Swiss farm for colonization by antibiotic-resistant enterobacterial isolates and to decipher the corresponding resistance mechanisms. A specific focus was made on $\beta$-lactams, particularly cephalosporins, since they are the most common antibiotics given for swine production. In addition, even if they are not part of the list of antibiotics given in that context, particular focus was placed on the occurrence of resistance to carbapenems (not given in animals), aminoglycosides, and polymyxins, as they represent crucial antibiotics in human medicine.

Rectal swabs were collected from healthy animals in a Swiss pig farm following the guidelines of SuisSano, a Swiss program for Animal Health from SuisAG since 2013 [5]. This program expects the farmer to record every antibiotic treatment of each pig, to use antimicrobial treatments as little as possible, and to strictly follow veterinary advice with respect to antibiotic usage.

\section{Material and Methods}

\subsection{Swabs Recovery}

The 81 fresh rectal swabs were recovered in M40 Amies media (Copan, Brescia, Italy) from healthy pigs on a farm in the Fribourg Canton (Switzerland). Swine were separated into ten stable boxes: three breeding sows with piglets in one box each, three boxes of weaning pigs (two with pigs from 18 to $20 \mathrm{~kg}$ and one from 9 to $10 \mathrm{~kg}$ ), three boxes of fattening pigs, and a single reproduction box with 10 sows. Pigs had no contact with the outside environment and were all born on-site. All data concerning the type of livestock per box are provided in Table 1.

\subsection{Selection of Resistant Isolates}

Rectal swabs were cultured two hours after collection in Luria-Bertani broth (LBB, Roth, Karlsruhe, Germany) overnight. After LBB enrichment, a $10 \mu \mathrm{L}$ loop of each broth was struck onto four different selective media, namely ChromID ESBL (bioMérieux, La Balme-La-Grotte, France) to select extended-spectrum $\beta$-lactamase (ESBL)-producing Gram-negatives, ChromID Carba Smart (bioMérieux) to select carbapenem-resistant Gramnegatives, home-made SuperAminoglycoside supplemented with amikacin $30 \mathrm{mg} / \mathrm{L}$ and gentamicin $30 \mathrm{mg} / \mathrm{L}$ to select pan-aminoglycoside-resistant Gram-negatives, and SuperPolymyxin supplemented with colistin $3.5 \mathrm{mg} / \mathrm{L}$ (EliTech Microbio, Signes, France) to select colistin-resistant Gram-negatives [6,7]. Grown colonies were tested by oxidase test (bioMérieux), and positive results were excluded as nonfermenting Gram-negative bacteria. 
Table 1. Rate and features of resistance isolates distribution in pig stables.

\begin{tabular}{|c|c|c|c|c|c|c|c|c|}
\hline Stable Number & Number of Pigs & $\begin{array}{c}\text { Number of } \\
\text { Resistant Isolates }\end{array}$ & Type of Livestock & Weight of Piglets & Clones Present & $\begin{array}{c}\text { Rate of Pigs Carrying } \\
\text { Resistant Strains }\end{array}$ & $\begin{array}{c}\text { Resistant } \\
\text { Determinant }\end{array}$ & $\begin{array}{l}\text { Co-Resistance } \\
\text { Phenotype }\end{array}$ \\
\hline \multirow[t]{2}{*}{1} & \multirow[t]{2}{*}{8} & \multirow[t]{2}{*}{7} & \multirow[t]{2}{*}{ Fattening } & \multirow[t]{2}{*}{40 to $42 \mathrm{~kg}$} & $\mathrm{~A}(n=6)$ & \multirow[t]{2}{*}{$87.50 \%$} & $b l a_{\mathrm{CTX}-\mathrm{M}-1}$ & $\begin{array}{l}\text { SUL, TET a }(n=1) ; \\
\text { SUL }(n=4) ; \\
\text { None }(n=1)\end{array}$ \\
\hline & & & & & $C(n=1)$ & & $b l a_{\mathrm{CTX}-\mathrm{M}-1}$ & $\begin{array}{l}\text { NAL, CIP, TET, SUL, } \\
\text { CHL, FLO }(n=1) \\
\text { SUL }(n=4) ; \text { NAL }\end{array}$ \\
\hline 2 & 8 & 5 & Fattening & 40 to $42 \mathrm{~kg}$ & $\mathrm{~A}(n=5)$ & $62.50 \%$ & $b l a_{\mathrm{CTX}-\mathrm{M}-1}$ & $\begin{array}{l}\text { CIP, TET, SUL, CHL, } \\
\text { FLO }(n=1)\end{array}$ \\
\hline 3 & 6 & 2 & Fattening & $102 \mathrm{~kg}$ & $\begin{array}{c}\mathrm{A}(n=1) \\
\mathrm{B} 1(n=1)\end{array}$ & $33.30 \%$ & $\begin{array}{l}b l a_{\text {CTX-M-1 }} \\
b l a_{\text {CTX-M-1 }}\end{array}$ & $\begin{array}{l}\text { SUL }(n=1) \\
\text { SUL }(n=1)\end{array}$ \\
\hline \multirow[t]{2}{*}{6} & \multirow[t]{2}{*}{20} & \multirow[t]{2}{*}{4} & Weaning & \multirow[t]{3}{*}{9 to $10 \mathrm{~kg}$} & $\mathrm{~A}(n=2)$ & \multirow[t]{3}{*}{$20 \%$} & $b l a_{\mathrm{CTX}-\mathrm{M}-1}$ & $\begin{array}{l}\text { SUL }(n=1) \text {; NAL, } \\
\text { CIP, SUL, TET, CHL, } \\
\text { FLO }(n=1) ;\end{array}$ \\
\hline & & & \multirow[t]{2}{*}{ Reproduction } & & $\begin{array}{c}\text { E. cloacae }(n=1) \\
\text { K. pneumoniae }(n=1) \\
\text { B1 }(n=7)\end{array}$ & & $\begin{array}{c}\mathrm{ND}^{\mathrm{d}} \\
\text { mgrB truncation } \\
\text { bla }_{\mathrm{CTX}-\mathrm{M}-1}\end{array}$ & $\begin{array}{c}\text { None }(n=1) \\
\text { None }(n=1) \\
\text { SUL, TET }(n=7)\end{array}$ \\
\hline 7 & 10 & 8 & & & $\mathrm{D}(n=1)$ & & $b l a_{\mathrm{CTX}-\mathrm{M}-1}$ & $\begin{array}{l}\text { GMI, KMN, TMN, } \\
\text { SUL, TET }(n=1)\end{array}$ \\
\hline 9 & 3 & 2 & Sow ${ }^{\mathrm{c}}$ with 3 piglets & & $\mathrm{B} 2(n=1)$ & $33 \%$ & $b l a_{\mathrm{TEM}-1}$ & $\begin{array}{c}\text { KMN, SUL, SXT, } \\
\text { TET }(n=1)\end{array}$ \\
\hline 10 & 3 & 1 & Sow ${ }^{d}$ with 3 piglets & & $\begin{array}{c}\mathrm{B} 1(n=1) \\
\mathrm{A}(n=1)\end{array}$ & $\begin{array}{l}33 \% \\
33 \%\end{array}$ & $\begin{array}{l}\text { bla } \\
\text { bla } \\
\text { CTХ-M-1 } 1 \\
\text { CTХ-1 }\end{array}$ & $\begin{array}{l}\text { SUL, TET }(n=1) \\
\text { SUL }(n=1)\end{array}$ \\
\hline
\end{tabular}

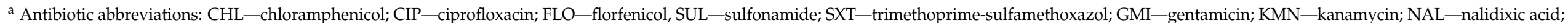

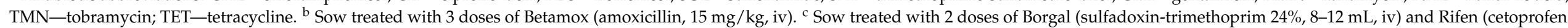
$10 \%, 3 \mathrm{~mL} / 100 \mathrm{~kg}$, iv). ${ }^{\mathrm{d}}$ Resistance to colistin, mechanism not determined. ND: not determined. 


\subsection{Identification and Phenotype of Resistance of Isolates}

Oxidase negative grown colonies were first tested by rapid in-house tests, namely the Rapid ESBL NP, Rapid Carba NP, or Rapid Polymyxin NP tests to confirm the resistance phenotype of each isolate [8-10]. Isolates were then identified at the species level by using the API 20E system (bioMérieux). In case of difficult biochemical identification, 16S rRNA PCR amplification was performed using boiled crude DNA extracts of the tested isolate, and with primers 8F (5'-AGAGTTTGATCCTGGCTCAG- $\left.3^{\prime}\right)$ and 1489R $\left(5^{\prime}\right.$-TACCTTGTTACGACTTCA-3 $\left.3^{\prime}\right)$ using an annealing temperature of $55^{\circ} \mathrm{C}$. Sequencing and Blast analysis of the sequence were performed using the NCBI website to identify the species. Disk diffusion assays (Bio-Rad, Crissier, Switzerland) on Mueller-Hinton agar (Roth) square plates were performed to evaluate the phenotype of each isolate and to determine the susceptibility to antibiotics, including amoxicillin, ticarcillin, piperacillin, temocillin, amoxicillin + clavulanic acid, ticarcillin + clavulanic acid, piperacillin + tazobactam, cephalothin, cefotaxime, ceftazidime, cefoxitin, cefepime, aztreonam, ertapenem, meropenem, imipenem, nalidixic acid, ciprofloxacin, tetracycline, tigecycline, chloramphenicol, fosfomycin, sulfonamide, sulfamethoxazole-trimethoprim, gentamicin, amikacin, tobramycin, and kanamycin, while susceptibility to colistin was tested by broth microdilution, according to EUCAST recommendations [11].

\subsection{Identification of $\beta$-Lactamase Genes}

Production of an ESBL was firstly assessed by using the Rapid ESBL, and then PCR amplification was performed on ESBL-positive isolates to identify the corresponding $\beta$ lactamase genes as previously described [12]. Sequencing of the obtained amplicons and in silico Blast analyses were performed to identify the exact nature of the $\beta$-lactamase gene (Microsynth, Balgach, Switzerland) [13].

\subsection{Genetic Subtyping}

Clonality of each isolate was evaluated by comparison of the DNA fingerprints obtained by pulsed-field gel electrophoresis (PFGE) according to CDC. Multilocus sequence typing (MLST) was performed on a representative isolate of each PFGE pattern. This method consists of the amplification of seven housekeeping genes ( $a d k, f u m C, g y r B, i c d, m d h$, $\operatorname{pur} A$, and $\operatorname{rec} A$ ) and sequencing. Identification of the allele of each gene is done by query of each sequence in PubMLST database (www.pubmlst.org) and MLST allelic profiles are determined according to Achtmann MLST scheme [14,15].

\subsection{Plasmid Characterization}

Plasmids carrying the $b l a_{\mathrm{CTX}-\mathrm{M}-1}$ were first extracted by using the Kieser method [16]. Purified plasmids from each isolate were then transformed in the recipient $E$. coli strain TOP10 by electroporation using a MicroPulser electroporator (Bio-Rad). Transformants were selected on a Luria-Bertani agar plate supplemented with $1 \mu \mathrm{g} / \mathrm{mL}$ of cefotaxime. PCR amplification of the bla $a_{\mathrm{CTX}-\mathrm{M}-1}$ gene was performed on the transformants in order to confirm the success of the transformation [12]. In order to identify the plasmid bearing the bla $_{\text {CTX-M-1 }}$ gene, PCR-based replicon typing (PBRT) using IncI1, IncF, IncHI1, and IncN primers were performed [17]. PCR was completed using DNA extracts from the E. coli transformants and in-house sequenced positive controls to determine the incompatibility group of the plasmids [18].

Plasmid extracts from E. coli transformants harboring representative plasmids bearing the gene $b l a_{\mathrm{CTX}-\mathrm{M}-1}$ were sequenced by Illumina for short reads and Nanopore for long reads. DNA library for Nanopore and Illumina sequencing was prepared using the NEBNext End Repair/dA-tailing module (Ipswich, MA, USA) and the ligation sequencing kit Nanopore SQK-LSK109 and Nextera XL library kit, respectively. The obtained plasmid data sequences in fast $Q$ were assembled and polished by CLC Main Genomic Workbench version 20.04 from Qiagen (Hilden, Germany). Annotation of the plasmid sequences was performed by CARD and analysis by PlasmidFinder $[19,20]$. 


\section{Results}

\subsection{Prevalence of $\beta$-Lactamase-Producing and Colistin-Resistant Enterobacterales}

A total of 39 ESBL-producing Enterobacterales was recovered from the 81 rectal swabs. Among them, 38 E. coli and a single E. cloacae were identified, all of them being positive for the $b l a_{\mathrm{CTX}-\mathrm{M}-1}$ gene. These ESBL producers were recovered from all the ten stable boxes from which samples had been recovered (Tables 1 and 2).

Table 2. Phenotype and genetic features associated with the ESBL-positive isolates.

\begin{tabular}{|c|c|c|c|c|c|c|c|}
\hline $\begin{array}{l}\text { Number } \\
\text { of Isolates }\end{array}$ & Species & $\begin{array}{l}\text { Phylogenic } \\
\text { Group }\end{array}$ & ST & Pulsotype & $\begin{array}{c}\text { Resistance } \\
\text { Determinants }\end{array}$ & $\begin{array}{l}\text { Incompatibility } \\
\text { Group of the Plasmid } \\
\text { Carrying bla } a_{\mathrm{CTX}-\mathrm{M}-1}\end{array}$ & $\begin{array}{l}\text { Coresistance on the } \\
\text { Plasmid Carrying } \\
\text { bla }_{\mathrm{CTX}-\mathrm{M}-1}\end{array}$ \\
\hline 22 & E. coli & A & ST10 & A & $b l a_{\mathrm{CTX}-\mathrm{M}-1}$ & Inc I1 & TET, SUL ${ }^{a}$ \\
\hline 12 & E. coli & A & ST10 & $\mathrm{B} 1^{\mathrm{b}}$ & $b l a_{\mathrm{CTX}-\mathrm{M}-1}$ & Inc I1 & TET, SUL \\
\hline 1 & E. coli & A & ST10 & $\mathrm{B} 2^{\mathrm{b}}$ & $b l a_{\text {CTX-M-1 }}$ & IncI1 & $\begin{array}{l}\text { GMI, KMN, TMN, } \\
\text { SUL, TET }\end{array}$ \\
\hline 1 & E. coli & A & ST744 & C & $b l a_{\mathrm{CTX}-\mathrm{M}-1}$ & Inc I1 & TET, SUL \\
\hline 1 & E. coli & A & ST34 & D & $b l a_{\text {CTX-M-1 }}$ & Inc I1 & TET, SUL \\
\hline 1 & E. coli & A & ST10 & E & bla ${ }_{\text {CTX-M-1 }}$ & Inc I1 & TET, SUL \\
\hline 1 & E. cloacae & & ND & & bla ${ }_{\text {CTX-M-1 }}$ & Inc I1 & TET, SUL \\
\hline
\end{tabular}

a Antibiotic abbreviations: SUL—sulfonamides; TET—-tetracycline; GMI—gentamicin; KMN—kanamycin; TMN—tobramycin; ND: not determined. ${ }^{\mathrm{b}}$ The pulsotype B was divided in B1 and B2 sub-pulsotypes according to co-resistance.

Three colistin-resistant Enterobacterales were recovered onto the SuperPolymyxin medium, two being K. pneumoniae and one being E. cloacae. One K. pneumoniae was found in a weaning pig of ca. $20 \mathrm{~kg}$, whereas the single $E$. cloacae and the other colistin-resistant $K$. pneumoniae were recovered from pigs living in the same stable (Table 2).

\subsection{Antimicrobial Resistance Features of Colistin-Resistant Isolates}

Colistin susceptibility testing showed that the isolates were resistant to colistin with MICs of 64,32 , and $128 \mathrm{mg} / \mathrm{L}$, respectively, but remained susceptible to $\beta$-lactams. PCR screening of $m c r-1$ to $m c r-9$ gave negative results for the three strains. Two K. pneumoniae isolates harbored a chromosomal mutation leading to a premature stop codon $\left(\mathrm{C} 28^{*}\right)$ in the $m g r B$ gene sequence, which was, therefore, the source of the acquired resistance as previously shown [6].

\subsection{Clonal Relationship}

PFGE analysis identified five different pulsotypes among the 38 ESBL-producing E. coli (Figure 1), the majority being from the sequence type (ST) ST10 and two isolates being ST34 and ST744, respectively, and also belonging to clonal complex CC10. Pulsotype A included 22 isolates, pulsotype B included 13 isolates, and pulsotype $C$ included 1 isolate, all belonging to ST10, while the 2 ST34 and ST744 isolates corresponded to pulsotypes $\mathrm{C}$ and D (Table 2). Pulsotype B was divided in sub-pulsotypes B1 (12 isolates) and B2 (1 isolate) according to the antibiotic co-resistances observed.

The two colistin-resistant K. pneumoniae showed a different pulsotype although both belonged to the same sequence type (ST76).

\subsection{Plasmid Analysis}

Transformants showing an ESBL phenotype were obtained from each pulsotype. PBRT analysis on the transformants showed that the $b a_{\mathrm{CTX}-\mathrm{M}-1}$ was always located on an IncI1type plasmid. Whole-plasmid sequencing was performed for those plasmids being the most prevalent, namely the two recovered from isolates belonging to pulsotypes A and B, respectively. Plasmid sequences of plasmids PCTX-M-1.A and PCTX-M-1.B1 have been deposited in GenBank under accession numbers MW978788 and MW978789, respectively. Sequence analysis revealed that both plasmids belong to the incompatibility group IncI1-I $\gamma$ and belonged to pMLST ST3 type. Those two plasmids (namely pCTX-M-1.A and pCTXM-1.B1) were 145,152 bp and 111,787 bp in size, respectively. Both plasmids revealed a similar backbone structure also found on the IncI1 reference plasmid R64 (GenBank 
accession number AP005147.1). Interestingly, these plasmids harbored the doc and phd toxin-antitoxin system genes, involved in plasmid maintenance, and the psiA and psiB SOS inhibition genes. Those plasmids bearing the $b l a_{\mathrm{CTX}} \mathrm{M}-1$ gene coharbored the sul 2 and tet $A$ genes, respectively encoding resistance to sulfonamides and tetracycline.

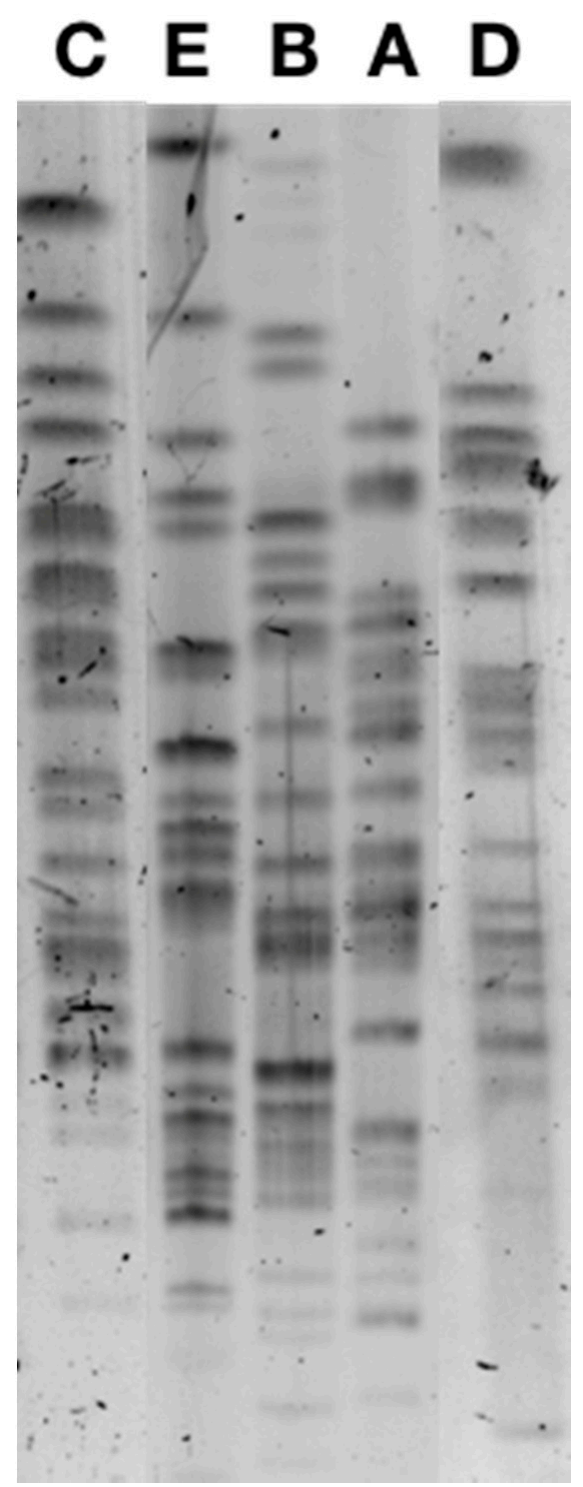

Figure 1. Pulsed-field gel electrophoresis of CTX-M-1-positive E. coli isolates corresponding to pulsotypes A, B, C, D, and E.

\section{Discussion}

In this study, a high prevalence of ESBL-producing Enterobacterales was found among pigs from a livestock farm in Switzerland, with $50 \%$ of the animals being colonized by ESBL-producing isolates, although only $4 \%$ were colonized with colistin-resistant $E$. coli or K. pneumoniae isolates. Neither carbapenem-resistant nor pan-aminoglycoside-resistant isolates could be recovered upon selection with respective selective media.

The rate of ESBL colonization was heterogeneous among the different stables (see Table 1).

This high rate of ESBL producers corresponded to an unexpected result. Indeed, such prevalence of ESBL-producing Enterobacterales was not expected in a farm with low antibiotic usage. To our knowledge, this is the first epidemiological report of ac- 
quired broad-spectrum cephalosporin resistance in a pig farm following such a struct antibiotic stewardship.

By contrast, the low rate of colistin-resistant isolates correlated with the lack of colistin usage for any purposes, including any food supplementation, and was in accordance with previous studies [12,21]. The three colistin-resistant isolates were negative for $\mathrm{mcr}$ genes, exhibiting nontransferable chromosomally encoded resistance mechanisms. It is worth noting that these three colistin-resistant K. pneumoniae were of ST76, an ST previously identified among NDM- or KPC-type carbapenemase producers recovered from humans in China [22-24]. This observation raises some concern about the epidemiology of such multidrug-resistant ST76 K. pneumoniae, strongly supporting the strict monitoring and prevention of its spread. In addition to a recent report, the low-level colistin resistance rate $(3.7 \%)$ evidenced in our study further highlights the benefit of banning colistin use in livestock [12].

Although evaluation of the clonality of the ESBL-producing E. coli revealed two major clones, they actually corresponded to a single sequence type, namely ST10, harboring a similar plasmid IncI1-I $\gamma$ harboring tet $A$ and sul2 antibiotic resistance genes in both cases (Table 2). The high cephalosporin resistance rate observed here was therefore mainly due to the spread of clonal strains rather than the dissemination of epidemic plasmids. It is noteworthy that very low rates of coresistance to fluoroquinolones were observed among those ESBL producers, in contrast to what is being observed among human isolates.

E. coli ST10 has been previously reported in animals and in humans [25]. IncI1-I $\gamma$-type plasmids harboring $b l a_{\mathrm{CTX}-\mathrm{M}-1}$ have already been identified not only in different foodproducing animals including broilers, poultry, and cattle, but also in the environment (rivers) and in both community patients and healthy people in Switzerland and around the world, being, therefore, a common E. coli background whose reservoir is hypothesized to be mainly animal $[17,26-28]$.

The high rate of CTX-M-1 producers (actually being commonly identified in animals) among fattening pigs and sows would suggest the presence of selective pressure in the farm. However, the absence of antibiotic usage in these fattening pigs did not support an antibiotic-related one. The maintenance of the CTX-M-1-encoding plasmid might, however, be explained by the presence of a toxin-antitoxin system [29-32]. The presence of toxinantitoxin systems on plasmids is known to be responsible for the postsegregational killing of daughter cells that do not contain the plasmid after the cell division completion [33]. On the other hand, the PsiAB proteins are known to allow a successful transfer of plasmid genetic information during conjugation by inhibiting the SOS system of the receptor bacteria, hence favoring their dissemination. Both systems indicate that the plasmid IncI1 pMLST3 identified here may be considered as a successful plasmid in terms of maintenance within a population and of dissemination. Nevertheless, our observations require further investigations to provide possible explanations about such an occurrence.

Author Contributions: Conceptualization, L.P. and O.P.; Methodology, L.P. and C.F.; Formal Analysis, L.P. and C.F.; Investigation, C.F.; Resources, L.P. and O.P.; Data Curation, L.P.; Writing-Original Draft Preparation, C.F.; Writing-Review and Editing, L.P. and P.N.; Visualization, L.P. and O.P.; Supervision, L.P. and P.N.; Project Administration, L.P.; Funding Acquisition, L.P. and P.N. All authors have read and agreed to the published version of the manuscript.

Funding: This work was funded by the University of Fribourg, the Swiss National Science Foundation [project FNS-407240_177381] and the Laboratoire Européen Associé INSERM «Emerging Antibiotic Resistance in Gram-negative Bacteria».

Data Availability Statement: The datasets used and/or analysed during the current study are available from the corresponding author on request.

Acknowledgments: We are grateful to Fritz Trachsel for his contribution in collecting animal samples. We are also very grateful to Pascal Toffel, Director of the Institut Grangeneuve, who offered us the opportunity to perform this study.

Conflicts of Interest: The authors declare no conflict of interest. 


\section{References}

1. Amusi, J.; Tamara, L.; Horton, R.; Winkler, A.S. Reconnecting for our future: The Lancet One Health commission. Lancet 2020, 395, 1469-1471. [CrossRef]

2. Colligon, P.J.; McEwen, S.A. One Health-Its importance in helping to better control antimicrobial resistance. Trop. Med. Infect. Dis. 2019, 4, 1-21.

3. Aarestrup, F.M.; Wegner, H.C.; Collignon, P. Resistane in bacteria of the food chain: Epidemiology and control strategies. Expert Rev. Anti-Infect. Ther. 2008, 6, 733-750. [CrossRef]

4. Bergšpica, I.; Kaprou, G.; Alexa, E.A.; Prieto, M.; Alvarez-Ordóñez, A. Extended spectrum $\beta$-lactamase (ESBL) Escherichia coli in pigs and pork meat in European Union. Antibiotics 2020, 9, 678. [CrossRef] [PubMed]

5. Suis, A.G. Available online: https://www.suisag.ch/fr/gesundheit/programme-sante-suissano (accessed on 22 February 2021).

6. Nordmann, P.; Jayol, A.; Poirel, L. Rapid detection of polymyxin resistance in Enterobacteriaceae. Emerg. Infect. Dis. 2016, 22, 1038-1043. [CrossRef]

7. Nordmann, P.; Mazé, A.; Culebras, E.; Dobias, J.; Jayol, A.; Poirel, L. A culture medium screening $16 S$ rRNA methylase-porducing pan-aminoglycoside resistant Gram-negative bacteria. Diag. Microbiol. Infect. Dis. 2018, 91, 118-122. [CrossRef]

8. Nordmann, P.; Jayol, A.; Poirel, L. An universal culture medium for screening polymyxin-resistant Gram-negative isoaltes. J. Clin. Microbiol. 2016, 54, 1395-1399. [CrossRef]

9. Dortet, L.; Poirel, L.; Nordmann, P. Rapid detection of ESBL-producing Enterobacteriaceae in blood culture. Emerg. Infect. Dis. 2015, 21, 504-507. [CrossRef]

10. Nordmann, P.; Poirel, L.; Dortet, L. Rapid detection of carbapenemase-producing Enterobacteriaceae. Emerg. Infect. Dis. 2012, 18, 1503-1507. [CrossRef]

11. European Comittee on Antimicrobial Susceptiblity Testing. In Breakpoint Tables for Interpretation of MICs and Zone Diameters; Version 10.0; European Comittee on Antimicrobial Susceptiblity Testing: Växjö, Sweden, 2020.

12. Fournier, C.; Aires-de-Sousa, M.; Nordmann, P.; Poirel, L. Occurrence of CTX-M-15 and MCR-1-producing Enterobacterales in pigs, Portugal; evidences of direct links with antibiotic selective pressure. Int. J. Antimicrob. Agents 2019, 55, 105802. [CrossRef]

13. National Center for Biotechnology Information. Available online: https://blast.ncbi.nlm.nih.gov/Blast.cg (accessed on 22 February 2021).

14. Centers for Disease Control and Prevention. Available online: https://www.cdc.gov/pulsenet/pdf/ecoli-shigella-salmonellapfge-protocol-508c.pdf (accessed on 22 February 2021).

15. Public Databases for Molecular Typing and Microbial Genome Diversity. Available online: https://pubmlst.org/bigsdb?db= pubmlst_ecoli_achtman_seqdef (accessed on 22 February 2021).

16. Kieser, T. Factors affecting the isolation of CCC DNA from Streptomyces lividans and Escherichia coli. Plasmid 1984, 12, 19-36. [CrossRef]

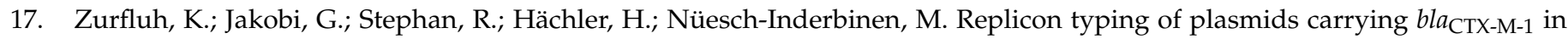
Enterobacteriaceae of animal, environmental and human origin. Front. Microb. 2014, 5, 555. [CrossRef]

18. Carattoli, A.; Bertini, A.; Villa, L.; Falbo, V.; Hopkins, K.L.; Threfall, E.J. Identification of plasmids by PCR-based replicon typing. Microbiol. Methods 2005, 63, 219-228. [CrossRef] [PubMed]

19. The Comprehensive Antibiotic Resistance Database. Available online: https:// card.mcmaster.ca (accessed on 22 February 2021).

20. Center for Genomic Epidemiology. Available online: https://cge.cbs.dtu.dk/services/PlasmidFinder/ (accessed on 22 February 2021).

21. Kieffer, N.; Aires-de-Sousa, M.; Nordmann, P.; Poirel, L. High rate of MCR-1-producing Escherichia coli and Klebsiella pneumoniae among pigs, Portugal. Emerg. Infect. Dis. 2017, 23, 2023-2029. [CrossRef] [PubMed]

22. Zhu, J.; Sun, L.; Ding, B.; Yang, Y.; Xu, X.; Liu, W.; Zhu, D.; Yang, F.; Zhang, H.; Hu, F. Outbreak of NDM-1-producing Klebsiella pneumoniae ST76 and ST37 isolates in neonates. Eur. J. Clin. Microbiol. Infect. Dis. 2016, 35, 611-618. [CrossRef]

23. Su, S.; Zhao, Y.; Yu, L.; Li, C.; Wang, Y.; Wang, Y.; Boa, M.; Fu, Y.; Li, C.; Zang, X. Outbreak of KPC-2 carbapenem-resistant Klebsiella pneumoniae ST76 and carbapenem-resistant K2 hypervirulent Klebsiella pneumoniae ST375 strains in Northeast China: Molecular and virulent characteristics. BMC Infect. Dis. 2020, 20, 472. [CrossRef]

24. Gong, X.; Zhang, J.; Su, J.; Fu, Y.; Bao, M.; Wang, Y.; Zhang, X. Characterization and epidemiology of carbapenem non-susceptible Enterobacteriacae isolates in the Eastern region of Heilongjiang Province, China. BMC Infect. Dis. 2018, 18, 417. [CrossRef]

25. Day, M.J.; Hopkins, K.L.; Warenham, D.W.; Toleman, M.A.; Elviss, N.; Randall, L.; Teale, C.; Cleary, P.; Wiuff, C.; Doumith, M.; et al. Extended-spectrum $\beta$-lactamase-producing Escherichia coli in human-derived and food chain-derived samples from England, Wales, and Scotland: An epidemiological surveillance and typing study. Lancet Infect. Dis. 2019, 19, 1325-1335. [CrossRef]

26. Zurfluh, K.; Wang, J.; Klumpp, J.; Nüesch-Inderbinden, M.; Fanning, S.; Stephan, R. Vertical transmission of highly simialr bla $a_{\mathrm{CTX}-\mathrm{M}-1}$-harboring IncI1 plasmids in Escherichia coli with different MLST types in the poultry production pyramid. Front. Microbiol. 2014, 5, 519. [CrossRef]

27. Geser, N.; Stephan, R.; Korczak, B.M.; Beutin, L.; Hächler, H. Molecular identification of extended-spectrum- $\beta$-lactamase from Enterobacteriaceae isolated from healthy human carriers in Switzerland. Antimicrob. Agents Chemother. 2012, 56, 1609-1612. [CrossRef] [PubMed] 
28. Valcek, A.; Roer, L.; Overballe-Petersen, S.; Hansen, F.; Bortolaia, V.; Leekitcharoenphon, P.; Korsgaard, H.B.; Seyfarth, A.M.; Hendriksen, R.S.; Hasman, H.; et al. IncI1 ST3 and IncI1 ST7 plasmids form CTX-M-1 producing Escherichia coli obtained from patients with bloodstream infections are closely related to plasmids form E. coli of animal origin. J. Antimicrob. Chemother. 2019, 74, 2171-2175. [CrossRef] [PubMed]

29. Smith, J.A.; Magnuson, R.D. Modular organisatzion of the PhD repressor/antitoxin protein. J. Bacteriol. 2004, 186, 2692-2698. [CrossRef]

30. Carattoli, A.; Villa, L.; Fortini, D.; García-Fernández, A. Contemporary IncI1 plasmids involved in the transmission and spread of antimicrobial resistance in Enterobacteriaceae. Plasmid 2018. [CrossRef] [PubMed]

31. Swiss Interior Federal Department Ordinance Regulation the Hygiene in Diary Production (OHyPL). RS 916.351.021.1 (State at the 8 December 2020). Available online: https:/ / www.fedlex.admin.ch/eli/cc/2005/824/fr (accessed on 22 February 2021).

32. Darphorn, T.S.; Bel, K.; Koenders-van Sint Anneland, B.B.; Brul, S.; Ter Kuile, B.H. Antibiotic resistance plasmid composition and architecture in Escherichia coli isolates form meat. Sci. Rep. 2021, 11, 2136. [CrossRef] [PubMed]

33. Rossolini, G.M.; D'Andrea, M.M.; Mugnaioli, C. The spread of CTX-M-type extended-spectrum $\beta$-lactamases. Clin. Microbiol. Infect. 2008, 14, 33-41. [CrossRef] [PubMed] 